\title{
Tuberculosis esofágica: informe de un caso
}

\section{A Case Report of Esophageal Tuberculosis}

Camilo A. Manrique M, MD, ${ }^{1}$ Diana Carolina Salinas G, MD, ${ }^{2}$ Julián D. Martínez, MD, ${ }^{3}$ Martín A. Garzón 0, MD, ${ }^{4}$ Geovanny Hernández C, MD, ${ }^{5}$ Natan Hormaza A, MD, ${ }^{4}$ Jorge I. Lizarazo R, MD, ${ }^{4}$ Juan Carlos Marulanda, MD, ${ }^{4}$ Juan Carlos Molano V, MD, ${ }^{4}$ Mario H. Rey T, MD. ${ }^{4}$

1 Residente de II año de Gastroenterología. Universidad del Rosario. Hospital Universitario de La Samaritana. Bogotá, Colombia

2 Residente III año medicina interna. Universidad del Rosario. Bogotá, Colombia

3 Profesor asociado, departamento de Medicina, Universidad Nacional de Colombia, Bogotá D.C. Colombia

${ }^{4}$ Gastroenterólogo. Servicio Gastroenterología Hospital Universitario de La Samaritana. Postgrado Gastroenterología Universidad del Rosario. Bogotá, Colombia

${ }^{5}$ Residente de I año de Gastroenterología. Universidad del Rosario. Hospital Universitario de La Samaritana. Bogotá, Colombia

Fecha recibido: 22-11-13 Fecha aceptado: $05-11-14$

\begin{abstract}
Resumen
Informamos el caso de un hombre inmunocompetente que consultó por cuadro de sangrado digestivo alto secundario a una úlcera esofágica de origen tuberculoso. El compromiso gastrointestinal por el Mycobacterium tuberculosis es raro aun en pacientes con altas prevalencias de enfermedad pulmonar y extrapulmonar; los sitios de mayor afectación son íleon terminal y el peritoneo, la enfermedad esofágica es exótica y generalmente es secundaria a la extensión proveniente de órganos vecinos infectados, como ganglios mediastinales y/o bronquios.

La clínica, las imágenes endoscópicas y radiológicas de la enfermedad esofágica suele simular una neoplasia maligna. La quimioterapia antituberculosa es la base del tratamiento, rara vez se requiere manejo quirúrgico.
\end{abstract}

\section{Palabras clave}

Esófago, tuberculosis, úlceras, sangrado gastrointestinal.

\begin{abstract}
\section{Keywords}

Esophageal tuberculosis, ulcers, gastrointestinal bleeding
\end{abstract}

We report the case of an immune-competent man with symptoms of upper gastrointestinal bleeding secondary to esophageal ulcers of tubercular origin. Gastrointestinal involvement from Mycobacterium tuberculosis is rare even in patients with numerous occurrences of pulmonary and extrapulmonary diseases. The most frequently affected sites are the terminal ileum and the peritoneum. Esophageal tuberculosis is exotic and is usually secondary to extension from neighboring infected organs such as the mediastinal nodes and/or the bronchi.

Clinical, endoscopic and radiological pictures of the esophageal disease often mimic malignancy. Tuberculosis treatment is the mainstay of treatment, and surgery is rarely required.

\section{PRESENTACIÓN DEL CASO}

Hombre de 86 años de edad, campesino, que ingresa por cuadro de hematemesis de dos días de evolución sin compromiso hemodinámico. Se realiza endoscopia de vías digestivas en la cual se observó en el tercio medio del esófago una lesión ulcerada sugestiva de neoplasia maligna, de $20 \mathrm{~mm}$ de diámetro, profunda y bordes levantados e indu- rados a la toma de biopsias, con estigmas de sangrado dados por presencia de coágulos de color negro adheridos a la lesión (figura 1). No se adviertió ningún otro tipo de lesión mucosa en estómago y duodeno; a pesar de la presencia de coágulos en la lesión y dada a su estabilidad hemodinámica se tomaron biopsias de la úlcera y se enviaron a patología.

El informe histopatológico mostró cambios de esofagitis granulomatosa de origen tuberculoso y la coloración de 
Ziehl-Nielsen señaló la presencia de abundantes bacilos ácido alcohol resistentes (figura 2).

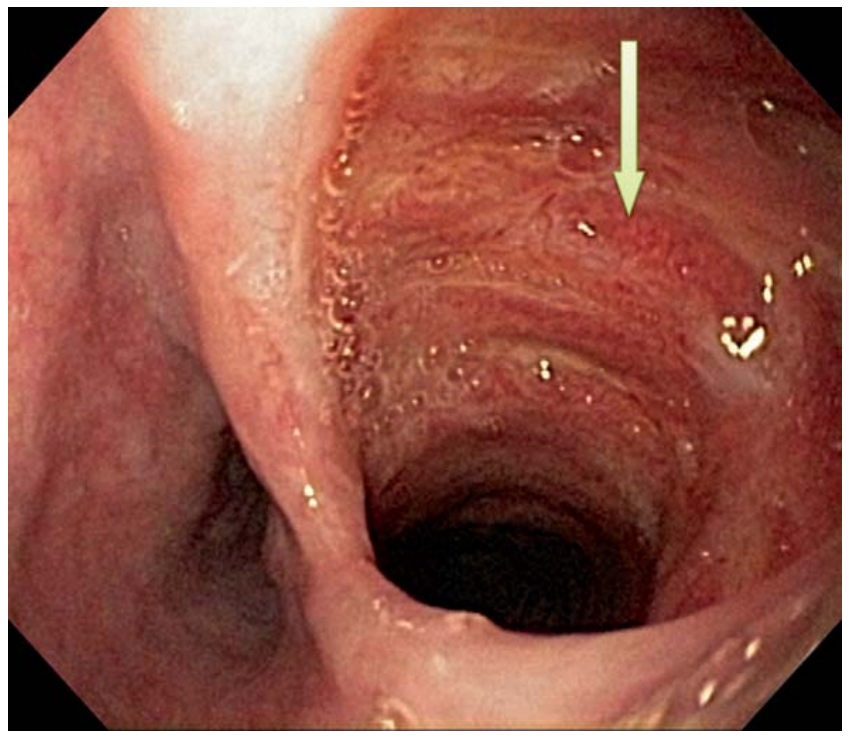

Figura 1. Imagen endoscópica de úlcera esofágica (flecha).

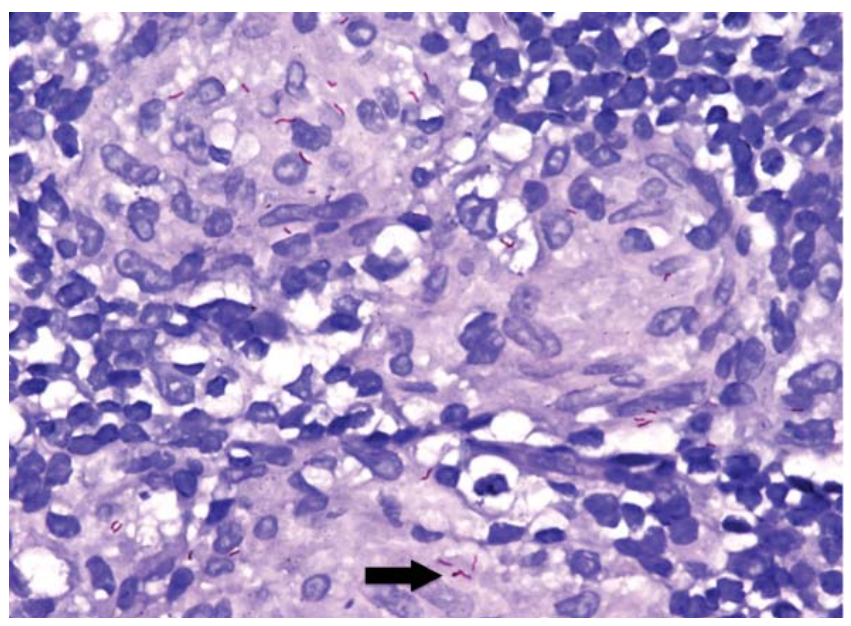

Figura 2. Microfotografía en donde se observan granulomas caseificantes y múltiples Mycobacterium tuberculosis (flecha).

Tanto el paciente como sus familiares negaron la coexistencia de síntomas respiratorios, se realizó radiografía de tórax en la cual se encuentran imágenes compatibles con tuberculosis miliar (figura 3), la prueba de ELISA para VIH fue negativa.

\section{COMENTARIOS}

La tuberculosis (TBC) es uno de los principales problemas de salud pública a nivel mundial, no solo de países en vía de desarrollo, sino de países industrializados. Cerca de un tercio de la población mundial (dos mil millones de personas) están infectadas por el Mycobacterium tuberculosis y aunque su incidencia ha disminuido, luego del pico epidemiológico del año 2003, aún sus cifras son alarmantemente altas pues se calcula en 8,8 millones/año de casos nuevos y 1,4 millones/año de muertes por esta causa, de acuerdo a la Organización Mundial de la Salud para 2010 (1).

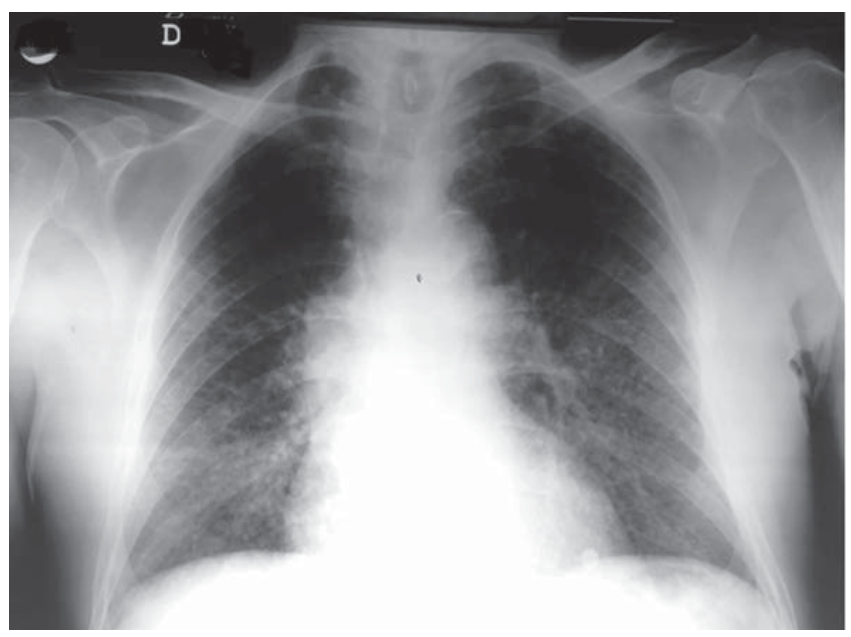

Figura 3. Rx tórax que muestran tuberculosis miliar.

La asociación a la infección por VIH se documenta en 1 de 14 casos nuevos, lo que sumado a la creciente resistencia antibiótica del bacilo, son consideradas las causas que explican el aumento de casos nuevos a nivel mundial (1).

La tuberculosis extrapulmonar representa $22 \%$ de todos los casos de tuberculosis, y más de la mitad de ellas se presenta en pacientes coinfectados con VIH (1).

En población sin $\mathrm{VIH}$, el compromiso extrapulmonar es más frecuente en los ganglios linfáticos (35\%), pleuras (20\%), huesos y articulaciones (10\%), tracto gastrointestinal (9\%), tuberculosis miliar (8\%), meningitis tuberculosa (5\%) y tuberculosis peritoneal (3\%) (2-4).

El compromiso esofágico por la tuberculosis es una entidad exótica que tiene dos formas de presentación: primaria y secundaria (5). La forma primaria es casi inexistente dada la falta de evidencia de esta, mientras que la forma secundaria aunque insólita, es la forma más frecuente y se origina por la extensión por contigüidad de estructuras mediastinales comprometidas como los ganglios linfáticos, bronquios y pleura. El contagio a través de esputo infectado deglutido y la diseminación linfangítica y hematógena de un foco pulmonar también han sido informados como causas de la infección esofágica (5).

El esófago cuenta con mecanismos de defensa que lo hacen menos susceptible a procesos infecciosos, entre estos están: la motilidad esofágica, la presencia del esfínter esofágico inferior y el estar revestido por un epitelio esca- 
moso en gran medida resistente a las infecciones; los anteriores mecanismos permiten una depuración más efectiva del bacilo tuberculoso; se corroboraron los hallazgos de necropsias en 18.049 en los cuales se observó compromiso esofágico en 0,15 a 0,14\% (3), lo que ubica al esófago como el octavo órgano en compromiso extrapulmonar.

Los síntomas de la tuberculosis esofágica son variados e inespecíficos, van desde el dolor abdominal, diarrea, vómito, anorexia, estreñimiento, pérdida de peso, sangrado gastrointestinal. Los síntomas están determinados principalmente por el compromiso en profundidad del órgano infectado que va desde la inflamación de la mucosa hasta úlceras profundas, la formación de lesiones exofíticas con compromiso de la luz (estenosis) principalmente en el tercio medio del órgano, por lo cual el principal diagnóstico diferencial es el carcinoma escamocelular (4).

La disfagia la presenta más de $90 \%$ de los pacientes, la cual es originada por ulceraciones de la mucosa, por lesiones exofíticas endoluminales, por fístulas, o por la compresión extrínseca por adenomegalias mediastinales o cervicales; el tercio medio del esófago es el sitio más afectado por la extensión de la infección desde adenopatías carinales frecuentemente encontradas en la formapulmonar de la enfermedad. Otras complicaciones encontradas son la formación de divertículos por tracción y la presencia de fístulas traqueoesofágicas $(3,6)$.

Diferentes estudios que han identificado factores de riesgo para compromiso extrapulmonar han documentado condiciones médicas concomitantes como neoplasias, corticoterapia, enfermedades del tejido conectivo, alcoholismo, falla renal, diabetes, embarazo; así mismo, las series de casos han demostrado que los hombres de raza negra tienen mayor susceptibilidad para progresión de la infección a enfermedad diseminada $(2,8,9)$.

Los hallazgos en la endoscopia digestiva y las radiografías esofágicas pueden ser variados, lo que incluye presencia de lesiones exofíticas que ocupan la luz del órgano, lesiones ulceradas profundas de gran extensión, presencia de divertículos por tracción y de fístulas con el árbol traqueobronquial. La tomografía de tórax, además de evaluar otras estructuras torácicas (pleura, pulmones, vértebras) determina la presencia de adenopatías mediastinales. La ultrasonografía endoscópica es un examen que gana terreno en el diagnóstico de la infección pues da información de gran precisión del compromiso de ganglios mediastinales y de otros órganos adyacentes además de permitir la toma de biopsias con aguja fina (10).

El diagnóstico definitivo de la infección se logra a través del aislamiento de bacilos en los medios de cultivo apropiado, la presencia de granulomas caseificantes y de bacilos ácido alcohol resistentes en las biopsias obtenidas de las lesiones, y la detección de fragmentos del genoma bacilar por técnicas de PCR las cuales han mostrado una sensibilidad de $80 \%$ y una especificidad de $100 \%$ en el diagnóstico de tuberculosis extrapulmonar $(3,7,11)$.

El tratamiento antituberculoso debe iniciarse en cuanto se confirme el diagnóstico ya que la adecuada respuesta lleva a mejoría de la sintomatología y resolución de la disfagia en los siguientes dos meses.

El tratamiento tetraconjugado recomendado está compuesto por isoniacida, rifampicina, pirazinamida y etambutol por dos meses y isoniacida y rifampicina por cuatro meses adicionales, esquema similar al que se emplea en el tratamiento de la tuberculosis pulmonar con tasas de éxito cercanas a 90\%; en pacientes seleccionados se requerirá la dilatación de las estenosis esofágica o la colocación de endoprótesis en el manejo de fístulas; rara vez se requiere manejo quirúrgico $(4,5)$.

\section{REFERENCIAS}

1. Global tuberculosis control: WHO report 2011. World Health Organization 2011.

2. Garcia JF, Alvarez HD, Lorenzo MV, Callejo A, Fernandez A, Sesma P. Extrapulmonary tuberculosis: epidemiology and risk factors. Enferm Infecc Microbiol Clin 2011; 29(7): 502-509.

3. Sharma S, Mohan A. Extrapulmonary tuberculosis. Indian J Med Res 2004; 20: 316-353.

4. Jain SK, Jain S, Jain M, Yaduvashi A. Esophageal tuberculosis: is it so rare? Report of 12 cases and review of the literature. Am J Gastroenterol 2002; 97: 287-91.

5. Sala A, Leibovich N, Souto G, Sabatini C, Brodersen C, Segal E. Tuberculosis esofágica: presentación de un caso y revisión de la literatura. Acta Gastroenterol Latinoam 2011; 41: 47-51.

6. Rathinam S, Kanagavel M, Sundaravadanan B, Santhosam R, Chandramohan S. Dysphagia due to tuberculosis. European Journal of Cardio-thoracic Surgery 2006; 30: 833-836.

7. Gómez C, Garrido A, Sansó J, Torres E. Tuberculosis esofágica en paciente inmunocompetente. Gastroenterol Hepatol 2003; 26(10): 643-5.

8. Fiske C, Griffin M, Erin H, Warkentin J, Lisa K, Arbogast K, Sterling T. Black race, sex, and extrapulmonary tuberculosis risk: an observational study. BMC Infectious Diseases 2010; 10: 16 .

9. Fitzgerald D, Sterling T, Haas D. Mycobacterium tuberculosis, extrapulmonary Tuberculosis. Mandell G, Benett J, Dolin R. Mandell, Douglas, and Bennett's Principles and Practice of Infectious Diseases. Cap 250. 7ma Ed. Elsevier; 2010. p. 3129-3163.

10. Hadlich E, Galperium B, Rizzon C. Esphageal ulcers caused by reactivation of ganglionary tuberculosis - a case report. Braz J infect Dis 2007; 11(2): 293-296.

11. Bresky G, Silva N, Madariaga J. Esofagitis tuberculosa en un paciente HIV positivo. Rev Med Chile 2007; 135: 13231326. 